\title{
Fucosylated glycoproteins as markers of liver disease
}

\author{
Anand Mehta* and Timothy M. Block \\ Drexel University College of Medicine, Department of Microbiology and Immunology, Drexel Institute for \\ Biotechnology and Virology, 3805 Old Easton Road, Doylestown, PA 18902, USA \\ Tel.: 215489 4905; Fax: 2154894920
}

\begin{abstract}
Changes in N-linked glycosylation are known to occur during the development of various diseases. For example, increased branching of oligosaccharides has been associated with cancer metastasis and has been correlated to tumor progression in human cancers of the breast, colon and melanomas. Increases in core fucosylation have also been associated with the development of hepatocellular carcinoma (HCC). Recently, changes in both the total serum glycome and the glycosylation of specific IgG molecules have been observed in people with liver fibrosis and cirrhosis. The mechanisms by which changes in glycosylation are observed and their use as biomarkers of disease will be discussed.
\end{abstract}

Keywords: Hepatitis B virus, Hepatitis C virus, fucosylation, liver fibrosis, liver cancer

\section{Introduction}

Infection of the liver with hepatitis B virus (HBV) and/or hepatitis $\mathrm{C}$ virus ( $\mathrm{HCV}$ ) is characterized by the ability to cause either acute infection that is frequently clinically inapparent or an unresolved, long term, chronic infection $[1,2]$. $10-40 \%$ of those chronically infected will develop either liver cirrhosis and/or primary hepatocellular carcinoma (HCC). Cirrhosis is a chronic disease of the liver where the normal liver architecture is replaced by fibrotic scar tissue, and is associated with an eventual decline of liver function. The development of cirrhosis is a pre-malignant condition and leads to an increased risk for the development of HCC [3-5]. Although there are many causative agents for cirrhosis, chronic viral infections of the liver by $\mathrm{HBV}$ and/or hepatitis $\mathrm{C}$ virus HCV are among the most common etiologies.

$\mathrm{HCC}$ is a cancer arising from the liver and is referred to as primary liver cancer. HCC is the fifth most common cancer in the world and is the 3rd leading cancer killer worldwide [6].The World Health Or-

\footnotetext{
*Corresponding author. E-mail: asm54@drexel.edu.
}

ganization (WHO) estimated that in 2005 there were 560,000 new cases of liver cancer worldwide, and a similar number of patients died as a result of this disease (http://www.who.int/en). The low survival rates have been attributed to the late diagnosis and poor therapeutic options [7].

\section{Methods for detecting liver disease}

For HBV and/or HCV infected patients, treatment decisions are based upon biochemical laboratory data, specifically hepatic transaminases, and more importantly the degree of hepatic inflammation and fibrosis on histological analysis [8]. For example, in individuals with HBV, advanced fibrosis and cirrhosis are considered justifications to begin antiviral therapy $[4,8,9]$. More importantly, the determination of hepatic fibrosis is critical to stage the severity of the liver disease in order to determine the prognosis and response to antiviral therapy [10]. It is thus extremely important to be able to determine the presence of significant fibrosis and cirrhosis in a manner that will allow for routine clinical monitoring. 
The gold standard for the staging of chronic liver disease is histopathological analysis, especially the determination of the amount of hepatic fibrosis [11]. The degree of liver injury in patients with chronic viral hepatitis is measured using a grading and staging system that are used to measure the degree of inflammation and fibrosis [12]. These are histological indicators that are classically associated with severity and progression of liver disease. However, biopsy is expensive, variable, and has low patient acceptance due to its inherent discomfort and potential risk for serious adverse outcomes. In addition, there is low concordance with the interpretation of the biopsy even among expert pathologists and suffers from sampling error, thereby, giving misleading results [13]. Alternative non-invasive tests for the measurement of hepatic fibrosis are urgently needed. Since the liver is a highly secretory organ, it has been reasoned that the circulation would contain selective molecules that, in their abundance or modification, would reflect the physiological state of the liver.

The progression of liver disease into liver cancer is monitored primarily by the use of serum levels of the oncofetal glycoprotein, alpha-fetoprotein (AFP), which is thought to be produced by transformed liver cells. However, AFP can be produced under many circumstances, including other liver diseases [14-16], and is not a definitive marker for the development of HCC. Analysis of the regulatory mechanisms of increased AFP synthesis in hepatic injury and in malignant transformations has been unable to distinguish elevation of AFP between HCC and chronic liver disease [17,18]. Hence the usefulness of AFP screening is limited and its use as a primary screen for HCC has been questioned [4].

\section{Changes in glycosylation associated with HCC}

The literature indicates that changes in glycosylation occur during the development of HCC. The most notable change is an increase in the level of core alpha 1,6 linked fucosylation of AFP [19,20]. In HCC and in testicular cancer, the glycosylation of AFP shifts from a simple biantennary glycan to an alpha 1,6 linked core fucosylated biantennary glycan. This change has been observed by both direct glycan sequencing of AFP and by increased reactivity of AFP with a variety of lectins that preferentially bind to fucose containing glycan [21]. The species of AFP that reacts preferentially with the lectin lens culinaris ( $\mathrm{LCH}$ ) is referred to as
AFP-L3. Several reports have clearly indicated that AFP-L3 is a more specific marker of HCC than is the total AFP protein level [22-24]. Indeed, AFP-L3, gained approval from the US Food and Drug administration (FDA) in 2005 as the only diagnostic assay for HCC.

In addition to the increases in fucosylation observed on AFP, other changes in N-linked glycosylation have also been observed. These change include the addition of bisecting N-Acetylglucosamine (GlcNAc) residues along with increased alpha 2,6 linked sialation [25]. These changes have been observed on a more global scale, rather than through the examination of a single glycoprotein.

Although the molecular mechanism of increased fucosylation in HCC is not clear [26-28], it is known that the increase is not restricted to AFP [29-31]. Results from several groups have indicated that other liver derived glycoproteins such as serotransferrin, and alpha 1 antitrypsin also become fucosylated with the development of HCC and a recent study has proposed that these glycoforms may be valuable biomarkers of HCC [31].

Recent work has identified changes in glycosylation that can be observed in the serum of people that correlate with the presence of HCC. This has been performed either on glycan analysis of whole serum [32], or serum that has been depleted of immunoglobulin [29,33]. In both circumstances, an increase in core fucosylation was observed with the development of HCC. To identify those proteins that had increased fucosylation, the fucosylated glycoproteins associated with sera from either pooled normal or pooled HCC positive individuals were extracted using fucose specific lectins and the proteome analyzed by either two dimensional gel electrophoresis (2DE) or by a multi-step LC MS/MS based methodology designed to identify fucosylated peptides (see Fig. 1) [29]. Using these methodologies it was determined that the fucosylated glycoforms of many liver derived glycoproteins are found in increased abundance in the serum following the development of primary liver cancer. Several of these glycoproteins have been analyzed in larger multi-cohort sample sets and show promise as biomarkers of liver cancer [29,33,34].

\section{Secretion of fucosylated proteins from the liver - loss of cell polarity?}

The reasons why there is an increase in fucosylated glycoforms in the serum of patients with liver cancer is a mystery but may be associated with loss of cell polarity. That is, many epithelial cells normally become po- 

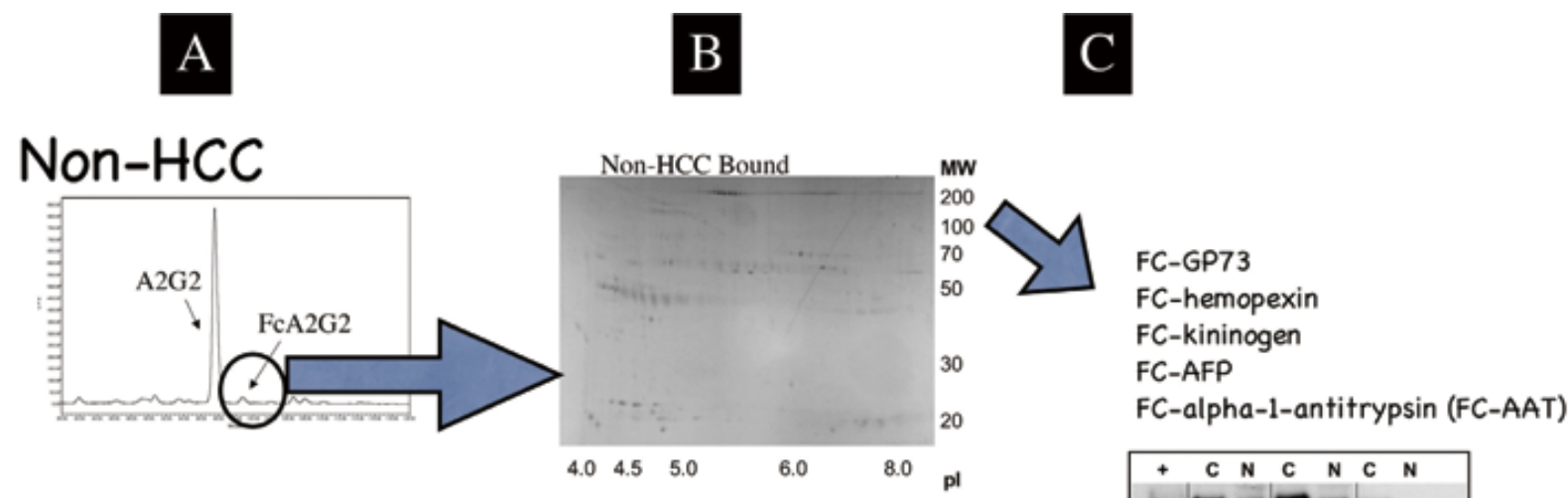

FC-alpha-1-antitrypsin (FC-AAT)
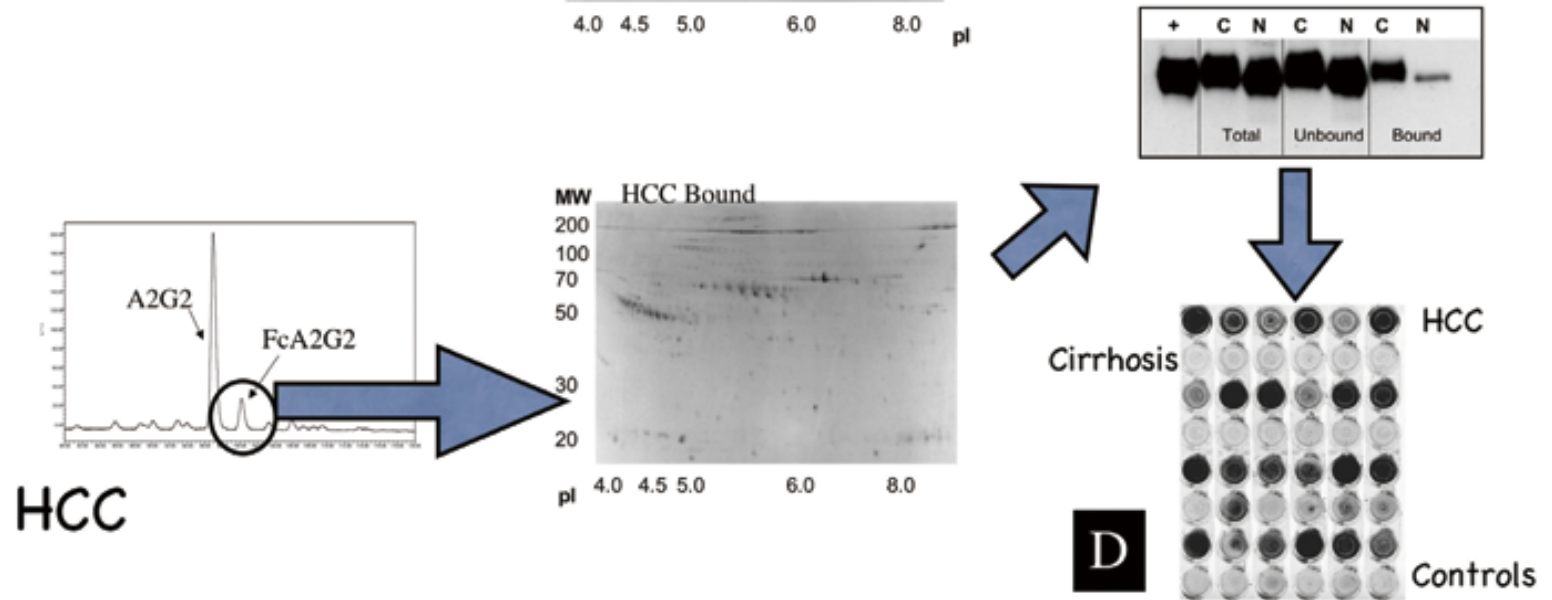

Fig. 1. Glycoproteomic platform for biomarker discovery: (A) Comparative Glycan analysis is performed to determine glycan change of interest. In the case of liver cancer the change is increased core fucosylation. (B) Glycan specific lectins (in this case to core fucose) are used to extract out those glycoproteins carrying the glycans of interest. Glycoprotein's can be analyzed either by various proteomic methodologies, 2DE is shown here. (C) Proteins identified by proteomics are validated by immunoblot of both total and lectin enriched fractions. In this particular gel the level of fucosylated $\square-1$ acid glycoprotein (AGP) in pooled serum from patients infected with HBV and positive for liver cancer (C) or just infected with HBV (N) as detected by immunoblotting is shown. From left to right: $(+)$ is the level of AGP in total serum purchased from Sigma Chemicals. The total lanes contain the level of AGP in the unfractionated Cancer and Normal serum. The unbound fraction contains the level of non fucosylated AGP in the cancer and normal serum while the bound fraction contains only the fucosylated AGP. (D) Eventually, a Lectin-ELISA is made for each protein identified that allows for screening of larger patient cohorts. A typical lectin-ELISA for fucosylated kininogen is shown.

larized, with at least two distinct plasma membrane surfaces. In the case of the intestinal epithelium, proteins are oriented either basolaterally (toward the blood) or apically (toward the lumen). Hepatocytes are unusual in that they polarize in three dimensions rather than as a two dimensional sheet. The basolateral surface is in contact with the blood, while the apical surfaces of the cells form the bile canaliculi Glycoproteins directed to these surfaces may be selective or even specific for the apical or basolateral surface, and thus maintenance of this polarity depends upon the continuous sorting of newly made proteins and membranes.

Recently, it has been suggested that fucosylation of $\mathrm{N}$-linked glycan within polarized hepatocytes directs glycoproteins to the apical surface and into the bile, and as a consequence, fucosylated glycoforms are normally rare in the blood, and are enriched in the bile [35]. Thus, if cancer cells become "depolarized", it is reasoned that fucosylated glycoforms would rise in abundance in the blood.

The sorting theory has appeal, and is consistent with levels of many fucosylated glycoproteins rising in the circulation of those with cancer. The theory has been examined for a few proteins, but must be confirmed. Even if fucosylation does play a role in sorting, that may not be the only function of fucosylation, and, even if correct, may not be the entire explanation for the increase in fucosylated proteins in those with liver cancer. For example, reports have indicated both an increase in the levels of several of the fucosyltransferase enzymes and transcripts in malignant and even non malignant tissue, surrounding the liver cancer and in 
A

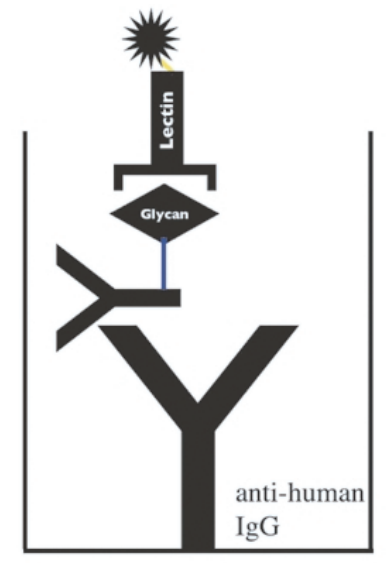

$\mathrm{B}$

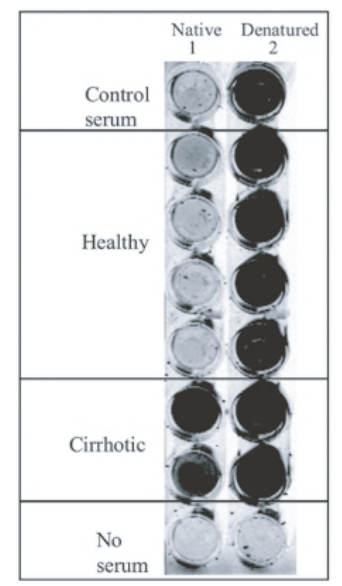

Fig. 2. Analysis of lectin reactive IgG as a function of protein folding via lectin FLISA. (A) Periodate oxidized mouse anti-human IgG was used as the capture antibody and the level of fucosylated protein determined by a biotin conjugated conjugated lectin (AAL) and detected using RDye ${ }^{\mathrm{TM}}$ 800 Conjugated Streptavidin and signal intensity measured using the Odyssey ${ }^{\mathrm{TM}}$ Infrared Imaging System. In all cases sample intensity was compared to commercially purchased human serum (Sigma Chemicals). (B) The reactivity of human IgG to the fucose binding lectin AAL was tested as a function of protein conformation. Purified human IgG from either four healthy subjects or two cirrhotic patients was captured using a monoclonal mouse anti-human IgG antibody and the level of fucosylated IgG detected using the fucose binding lectin AAL. For each sample, IgG was either left in the native state, or reduced and alkalyated before analysis. For FLISA, left column is the lectin-FLISA on IgG in a native state; right column is the lectin-FLISA on IgG after the protein has been denatured. As this Figure shows, while only IgG from patients with cirrhosis are reactive to the fucose binding lectin AAL in a native state, all IgG molecules are reactive to the fucose binding lectin AAL when denatured. Samples without serum were used to measure reactivity of lectin to capture antibody.

the level of fucose substrate, UDP-L-Fucose, required for fucosylation $[26,27,36]$. Thus, these increases, and not depolarization, could account for the elevated core fucosylation of cancer cell produced glycoproteins.

\section{Changes in the glycosylation of IgG as a function of liver fibrosis}

Recently it has been determined that the glycosylation of human immunoglobulin $\mathrm{G}$ ( $\mathrm{IgG}$ ) molecules reactive to the heterophilic alpha-gal epitope changes with the development of cirrhosis [37]. Heterophilic alpha-gal antibodies are naturally occurring antibodies that constitute $1 \%$ of total serum $\operatorname{IgG}$ and interact with a specific sugar linkage on glycolipids and glycoproteins [38-40]. This sugar linkage (Gal $\alpha-1-$ $3 \mathrm{Gal} \beta 1-3-\mathrm{GlcNAc-R}$ ), referred to as the alpha-gal epitope, is absent in humans but is abundantly synthesized by bacteria, yeast and nonprimate mammals and in New World monkeys, which instead produce antibodies to this structure (the alpha-gal antibody) in large amounts. It has long been believed that alpha-gal antibodies control the level of Enterobacteriaceae, which are commonly found as a normal part of the human and animal gut flora and express the alpha-gal epitope.
In addition to the change in glycosylation, greatly increased binding of several fucose-binding lectins to alpha-gal specific IgG has been observed. [37]. This is paradoxical, as the change observed on alpha-gal IgG was a reduction in galactosylation and the level of fucose remained constant. Surprisingly, while human immunoglobulin (in general) contains predominantly core fucosylated glycans, lectins specific to core fucose bind poorly to native IgG from healthy individuals. In contrast, native IgG molecules from subjects with cirrhosis bind greater amounts of fucose binding lectin. Strong lectin binding from all subjects does occur following denaturation of the antibody, suggesting a conformational change in IgG with the development of cirrhosis (see Fig. 2).

These two surprising findings, the alteration in the glycosylation of heterophilic alpha-gal antibodies and the greater association with fucose binding lectins, has lead to the development of a simple method to monitor liver fibrosis and cirrhosis. In a $300+$ patient coded study, this assay was shown to be able to differentiate severe fibrosis and cirrhosis from mild fibrosis with positive predictive value of $97 \%$ [37].

While it is currently unclear why changes in the glycosylation of alpha gal IgG occur with the development of fibrosis/cirrhosis, an exciting possibility is that it is 


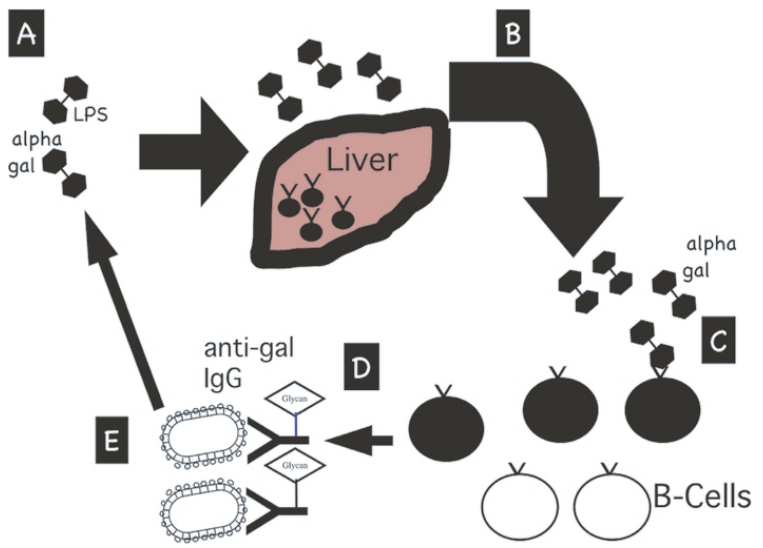

Fig. 3. Proposed model for the role of alpha-gal antibodies in the development of lver disease. (A) Bacterial products that are normally produced everyday by the death and destruction of gut flora are normally cleared by the liver. This includes bacterial toxins such as Lipopolysaccharide (LPS), which contain the alpha-gal sugar epitope as well as other bacterial products that contain the alpha-gal epitope. (B) In patients with liver disease, the clearance of these bacterial products is compromised and hence, the bacterial antigens can either bypass the liver (or enter the liver) and through (C) interaction with alpha-gal specific B cells, lead to the generation of a humoral immune response (D). This immune response could lead to more bacterial destruction and bacterial products in the serum (E). Thus, the constant exposure to bacterial products such as LPS could lead to greater levels of inflammation and the acceleration of liver disease. See text for more detail.

the result of immune stimulation. That is, it has been shown that a decrease in galactosylation of IgG is associated with repeated immunization and response to a specific antigen [41]. Surprisingly, this is the same alteration that we have observed and it strongly suggests that there may be clonal expansion of a specific set of $\mathrm{B}$ cells, presumable those that secrete heterophilic alphagal antibodies. It has been recently reported that $\mathrm{IgG}$ mediates pro- and anti-inflammatory activities through the engagement of its $\mathrm{Fc}$ domain $(\mathrm{Fc})$ with distinct $\mathrm{Fc}$ receptors (FcgRs). One type of interaction generates a pro-inflammatory effect while other interactions generate anti-inflammatory effects. The type of interaction is dependent upon the presence of terminal sialic acid residues on the $\mathrm{N}$-linked glycan present on the IgG molecule [42]. IgG molecules containing terminal sialic acid molecules lead to anti-inflammatory responses while $\operatorname{IgG}$ molecules lacking terminal sialic acid lead to a pro-inflammatory response. It is hypothesized here that the shift to N-linked glycan lacking terminal galactose residues, which prevents the further addition of sialic acid molecules, is indicative of antibody response to bacteria. An interesting hypothesis is how this could influence pathology. As shown in
Fig. 4, bacterial products that are normally produced everyday by the death and destruction of gut flora are cleared by the liver sinusoids. This includes bacterial toxins such as Lipopolysaccharide (LPS), which contains the alpha-gal sugar epitope and has been implicated as playing a role in the development of liver fibrosis [43-45]. However, in patients with liver disease, the clearance of these bacterial products is compromised and the bacterial antigens can either bypass the liver (or enter the liver) and through interaction with alpha-gal specific B cells, lead to the generation of a humoral immune response directed towards the alpha-gal epitope. This immune response could lead to more bacterial destruction and bacterial products in the serum. Thus, the constant exposure to bacterial products such as LPS could lead to greater levels of inflammation and the acceleration of liver disease. Thus, the change in the glycosylation of alpha- gal IgG molecules may act not only as a biomarker of liver fibrosis, but may play a major role in the disease itself. This is currently under investigation.

\section{Conclusion}

Changes in N-linked glycosylation have long been associated with the development of disease [46-50]. In the case of liver cancer, it has been clearly shown that an increase in core fucosylation is associated with the development of disease, in both animal models and in people [19,20,22-24,29,33,51,52]. This finding has lead to the only diagnostic marker of HCC approved by the U.S Food and Drug Administration (FDA). However, for HCC, this may represent just the tip of the iceberg. As many more glycoproteins with altered glycans are identified, a marker that can identify early liver cancer with $100 \%$ sensitivity and $100 \%$ specificity may soon be at hand.

Recent findings that changes in glycosylation of alpha-gal occur with the development of liver fibrosis may help in the development of a simple, inexpensive and non invasive assay and may provide insights into the development of liver disease.

\section{Acknowledgements}

This work was supported by grant UO1 CA08495106 from the National Cancer Institute (NCI) Early Detection Research Network (EDRN), grant R01 CA120206-01 also from the NCI, the Hepatitis B Foundation, and an appropriation from The Commonwealth of Pennsylvania. 


\section{References}

[1] A. Lok and B. McMahon, Chronic hepatitis B, Hepatology 34(6) (2001), 1225-1241.

[2] A.S. Lok, Natural history and control of perinatally acquired hepatitis B virus infection, Dig Dis 10(1) (1992), 46-52.

[3] J.A. Marrero, Hepatocellular carcinoma, Curr Opin Gastroenterol 22(3) (2006), 248-253.

[4] M. Sherman, Hepatocellular carcinoma: epidemiology, risk factors, and screening, Semin Liver Dis 25(2) (2005), 143 154.

[5] B.S. Anand, Cirrhosis of liver, West J Med 171(2) (1999), 110-115.

[6] T.M. Block, A.S. Mehta, C.J. Fimmel and R. Jordan, Molecular viral oncology of hepatocellular carcinoma, Oncogene 22(33) (2003), 5093-5107.

[7] A.M. Di Bisceglie, R.L. Carithers, Jr. and G.J. Gores, Hepatocellular carcinoma, Hepatology 28(4) (1998), 1161-1165.

[8] S.L. Fung and A.S. Lok, Management of hepatitis B patients with antiviral resistance, Antivir Ther 9(6) (2004), 1013-1026.

[9] Y.O. Kweon, Z.D. Goodman, J.L. Dienstag, E.R. Schiff, N.A. Brown, E. Burchardt, R. Schoonhoven, D.A. Brenner and M.W. Fried, Decreasing fibrogenesis: an immunohistochemical study of paired liver biopsies following lamivudine therapy for chronic hepatitis B, J Hepatol 35(6) (2001), 749-755.

[10] Y.F. Liaw, Results of lamivudine trials in Asia, J Hepatol 39(Suppl 1) (2003), S111-115.

[11] K. Ishak, A. Baptista, L. Bianchi, F. Callea, J. De Groote, F. Gudat, H. Denk, V. Desmet, G. Korb, R.N. MacSween et al., Histological grading and staging of chronic hepatitis, $J$ Hepatol 22(6) (1995), 696-699.

[12] R.G. Knodell, K.G. Ishak, W.C. Black, T.S. Chen, R. Craig, N. Kaplowitz, T.W. Kiernan and J. Wollman, Formulation and application of a numerical scoring system for assessing histological activity in asymptomatic chronic active hepatitis, Hepatology 1(5) (1981), 431-435.

[13] R.J. Fontana and A.S. Lok, Noninvasive monitoring of patients with chronic hepatitis C, Hepatology 36(5 Suppl 1) (2002), S57-64.

[14] M.E. Alpert, J. Uriel and B. de Nechaud, alpha fetogloblin in the diagnosis of human hepatoma, $N$ Engl J Med 278 (1968), 984-986.

[15] E. Ruoslahti, M. Salaspuro, H. Pihko, L. Andersson and M. Seppala, Serum alpha-fetoprotein: diagnostic significance in liver disease, Br Med J 2(918) (1974), 527-529.

[16] A.M.a.J.H.H. Di Bisceglie, Elevations in serum alphafetoprotein levels in patients with chronic hepatitis B, Cancer 64(10) (1989), 2117-2120.

[17] H. Nakabayashi, K. Watanabe, A. Saito, A. Otsuru, K. Sawadaishi and T. Tamaoki, Transcriptional regulation of alpha-fetoprotein expression by dexamethasone in human hepatoma cells, J Biol Chem 264(1) (1989), 266-271.

[18] K. Sawadaishi, T. Morinaga and T. Tamaoki, Interaction of a hepatoma-specific nuclear factor with transcription-regulatory sequences of the human alpha-fetoprotein and albumin genes, Mol Cell Biol 8(12) (1988), 5179-5187.

[19] J. Breborowicz, A. Mackiewicz and D. Breborowicz, Microheterogeneity of alpha-fetoprotein in patient serum as demonstrated by lectin affino-electrophoresis, Scand J Immunol 14(1) (1981), 15-20.

[20] J. Miyazaki, Y. Endo and T. Oda, Lectin affinities of alphafetoprotein in liver cirrhosis, hepatocellular carcinoma and metastatic liver tumor, Acta Hepatol Jpn 22 (1981), 15591568 .
[21] P.J. Johnson, T.C. Poon, N.M. Hjelm, C.S. Ho, C. Blake and S.K. Ho, Structures of disease-specific serum alphafetoprotein isoforms, Br J Cancer 83(10) (2000), 1330-1337.

[22] K. Taketa, E. Ichikawa, H. Taga and H. Hirai, Antibodyaffinity blotting, a sensitive technique for the detection of alpha-fetoprotein separated by lectin affinity electrophoresis in agarose gels, Electrophoresis 6 (1985), 492-497.

[23] K. Taketa, C. Sekiya, M. Namiki, K. Akamatsu, Y. Ohta, Y. Endo and K. Kosaka, Lectin-reactive profiles of alphafetoprotein characterizing hepatocellular carcinoma and related conditions, Gastroenterology 99(2) (1990), 508-518.

[24] K. Shiraki, K. Takase, Y. Tameda, M. Hamada, Y. Kosaka and T. Nakano, A clinical study of lectin-reactive alpha-fetoprotein as an early indicator of hepatocellular carcinoma in the followup of cirrhotic patients, Hepatology 22(3) (1995), 802-807.

[25] W. Morelle, C. Flahaut, J.C. Michalski, A. Louvet, P. Mathurin and A. Klein, Mass spectrometric approach for screening modifications of total serum N-glycome in human diseases: application to cirrhosis, Glycobiology 16(4) (2006), 281-293.

[26] K. Noda, E. Miyoshi, N. Uozumi, C.X. Gao, K. Suzuki, N. Hayashi, M. Hori and N. Taniguchi, High expression of alpha1-6 fucosyltransferase during rat hepatocarcinogenesis, Int J Cancer 75(3) (1998), 444-450.

[27] W.L. Hutchinson, M.Q. Du, P.J. Johnson and R. Williams, Fucosyltransferases: differential plasma and tissue alterations in hepatocellular carcinoma and cirrhosis, Hepatology 13(4) (1991), 683-688.

[28] E. Miyoshi, K. Noda, Y. Yamaguchi, S. Inoue, Y. Ikeda, W. Wang, J.H. Ko, N. Uozumi, W. Li and N. Taniguchi, The alpha1-6-fucosyltransferase gene and its biological significance, Biochem Biophys Acta 1473(1) (1999), 9-20.

[29] M.A. Comunale, M. Lowman, R.E. Long, J. Krakover, R. Philip, S. Seeholzer, A.A. Evans, H.W.L. Hann, T.M. Block and A.S. Mehta, Proteomic analysis of serum associated fucosylated glycoproteins in the development of primary hepatocellular carcinoma, Journal of Proteome Research 6(5) (2006), 308-315.

[30] K. Yamashita, N. Koide, T. Endo, Y. Iwaki and A. Kobata, Altered glycosylation of serum transferrin of patients with hepatocellular carcinoma, J Biol Chem 264(5) (1989), 24152423.

[31] A. Naitoh, Y. Aoyagi and H. Asakura, Highly enhanced fucosylation of serum glycoproteins in patients with hepatocellular carcinoma, J Gastroenterol Hepatol 14(5) (1999), 436-445.

[32] N. Callewaert, H. Van Vlierberghe, A. Van Hecke, W. Laroy, J. Delanghe and R. Contreras, Noninvasive diagnosis of liver cirrhosis using DNA sequencer based total serum protein glycomics, Nature Medicine 10(4) (2004), 429-434.

[33] T.M. Block, M.A. Comunale, M. Lowman, L.F. Steel, F.R. Romano, C. Fimmel, B.C. Tennant, W.T. London, A.A. Evans, B.S. Blumberg, R.A. Dwek, T.S. Mattu and A.S. Mehta, Use of targeted glycoproteomics to identify serum glycoproteins that correlate with liver cancer in woodchucks and humans, Proc Natl Acad Sci USA 102(3) (2005), 779-784.

[34] R.R. Drake, E.E. Schwegler, G. Malik, J. Diaz, T. Block, A. Mehta and O.J. Semmes, Lectin Capture Strategies Combined with Mass Spectrometry for the Discovery of Serum Glycoprotein Biomarkers, Mol Cell Proteomics 5(10) (2006), 19571967.

[35] T. Nakagawa, N. Uozumi, M. Nakano, Y. Mizuno-Horikawa, N. Okuyama, T. Taguchi, J. Gu, A. Kondo, N. Taniguchi and E. Miyoshi, Fucosylation of N-glycans regulates the secretion of hepatic glycoproteins into bile ducts, J Biol Chem 281(40) (2006), 29797-29806. 
[36] K. Moriwaki, K. Noda, T. Nakagawa, M. Asahi, H. Yoshihara, N. Taniguchi, N. Hayashi and E. Miyoshi, A high expression of GDP-fucose transporter in hepatocellular carcinoma is a key factor for increases in fucosylation, Glycobiology 17(12) (2007), 1311-1320.

[37] A.S. Mehta, R.E. Long, M.A. Comunale, M. Wang, L. Rodemich, J. Krakover, R. Philip, J.A. Marrero, R.A. Dwek and T.M. Block, Increased levels of galactose-deficient anti-Gal immunoglobulin $\mathrm{G}$ in the sera of hepatitis $\mathrm{C}$ virus-infected individuals with fibrosis and cirrhosis, J Virol 82(3) (2008), 1259-1270.

[38] U. Galili, E.A. Rachmilewitz, A. Peleg and I. Flechner, A unique natural human IgG antibody with anti-alpha-galactosyl specificity, J Exp Med 160(5) (1984), 1519-1531.

[39] U. Galili, Evolution and pathophysiology of the human natural anti-alpha-galactosyl IgG (anti-Gal) antibody, Springer Semin Immunopathol 15(2-3) (1993), 155-171.

[40] U. Galili, F. Anaraki, A. Thall, C. Hill-Black and M. Radic, One percent of human circulating B lymphocytes are capable of producing the natural anti-Gal antibody, Blood 82(8) (1993), 2485-2493.

[41] G.C. Lastra, S.J. Thompson, A.S. Lemonidis and C.J. Elson, Changes in the galactose content of IgG during humoral immune responses, Autoimmunity 28(1) (1998), 25-30.

[42] Y. Kaneko, F. Nimmerjahn and J.V. Ravetch, Antiinflammatory activity of immunoglobulin $\mathrm{G}$ resulting from $\mathrm{Fc}$ sialylation, Science 313(5787) (2006), 670-673.

[43] J. Ewaschuk, R. Endersby, D. Thiel, H. Diaz, J. Backer, M. Ma, T. Churchill and K. Madsen, Probiotic bacteria prevent hepatic damage and maintain colonic barrier function in a mouse model of sepsis, Hepatology 46(3) (2007), 841-850.

[44] S.L. Friedman, A deer in the headlights: BAMBI meets liver fibrosis, Nat Med 13(11) (2007), 1281-1282.
[45] E. Seki, S. De Minicis, C.H. Osterreicher, J. Kluwe, Y. Osawa, D.A. Brenner and R.F. Schwabe, TLR4 enhances TGF-beta signaling and hepatic fibrosis, Nat Med 13(11) (2007), 13241332.

[46] S. Kellokumpu, R. Sormunen and I. Kellokumpu, Abnormal glycosylation and altered Golgi structure in colorectal cancer: dependence on intra-Golgi pH, FEBS Lett 516(1-3) (2002), 217-224.

[47] R. Peracaula, G. Tabares, L. Royle, D.J. Harvey, R.A. Dwek, P.M. Rudd and R. de Llorens, Altered glycosylation pattern allows the distinction between prostate-specific antigen (PSA) from normal and tumor origins, Glycobiology 13(6) (2003), $457-470$.

[48] J. Taylor-Papadimitriou, J. Burchell, D.W. Miles and M. Dalziel, MUC1 and cancer, Biochim Biophys Acta 1455(2-3) (1999), 301-313.

[49] Y.J. Kim and A. Varki, Perspectives on the significance of altered glycosylation of glycoproteins in cancer, Glycoconj $J$ 14(5) (1997), 569-576.

[50] J.W. Dennis, M. Granovsky and C.E. Warren, Glycoprotein glycosylation and cancer progression, Biochem Biophys Acta 1473(1) (1999), 21-34.

[51] P.C. Kelleher, C.J. Walters, B.D. Myhre, B.C. Tennant, J.L. Gerin and P.J. Cote, Altered glycosylation of alphafetoprotein in hepadnavirus-induced hepatocellular carcinoma of the woodchuck, Cancer Lett 63(2) (1992), 93-99.

[52] A.B. Ajdukiewicz, P.C. Kelleher, E.L. Krawitt, C.J. Walters, P.B. Mason, R.S. Koff and L. Belanger, Alpha-fetoprotein glycosylation is abnormal in some hepatocellular carcinoma, including white patients with a normal alpha-fetoprotein concentration, Cancer Lett 74(1-2) (1993), 43-50. 


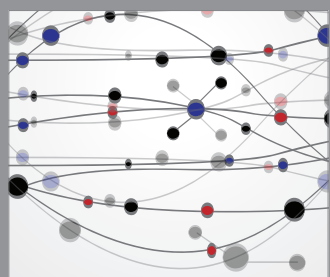

The Scientific World Journal
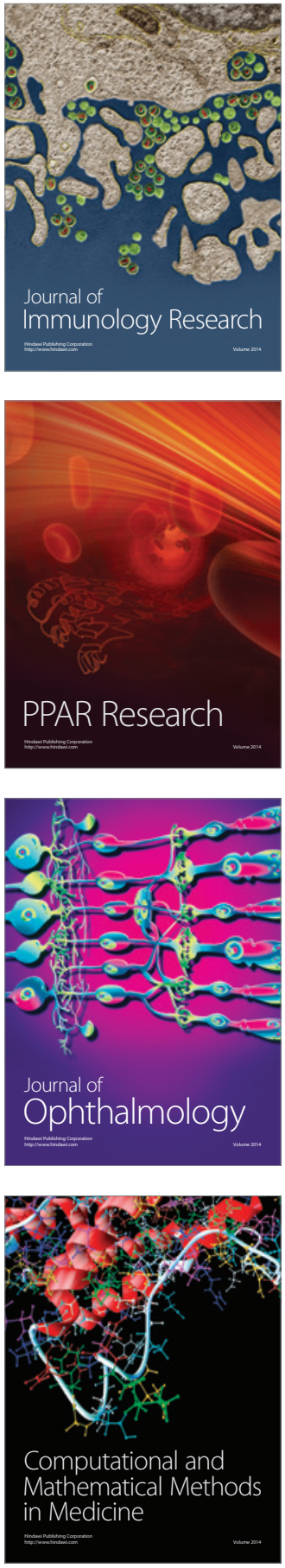

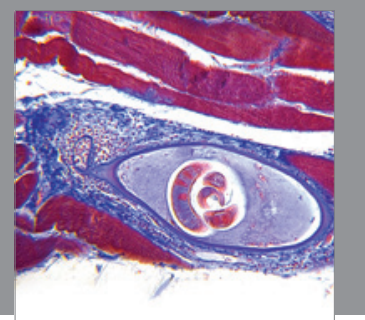

Gastroenterology

Research and Practice
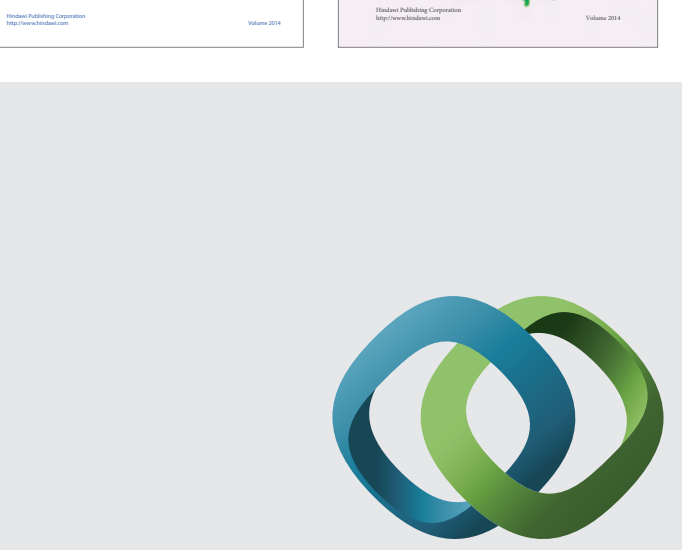

\section{Hindawi}

Submit your manuscripts at

http://www.hindawi.com
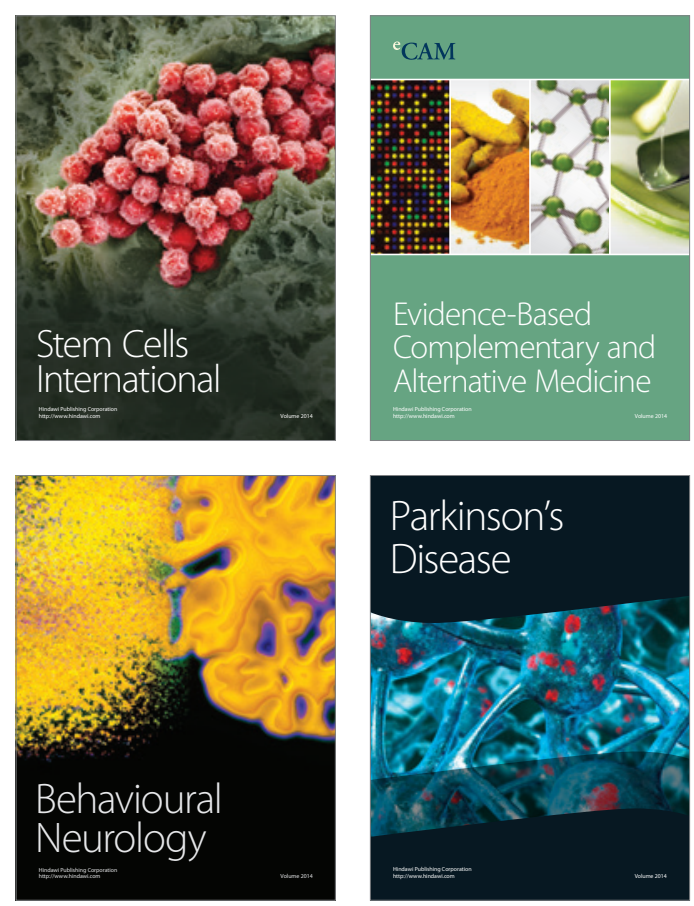

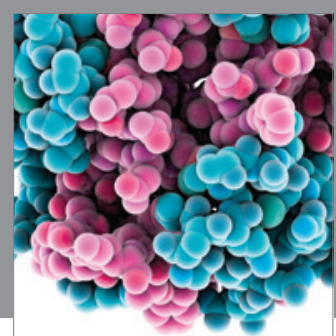

Journal of
Diabetes Research

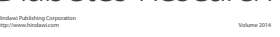

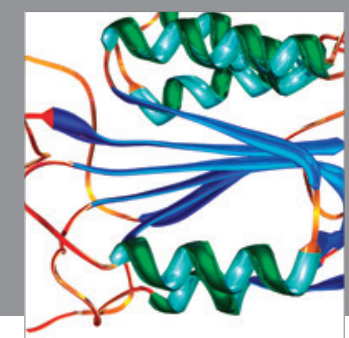

Disease Markers
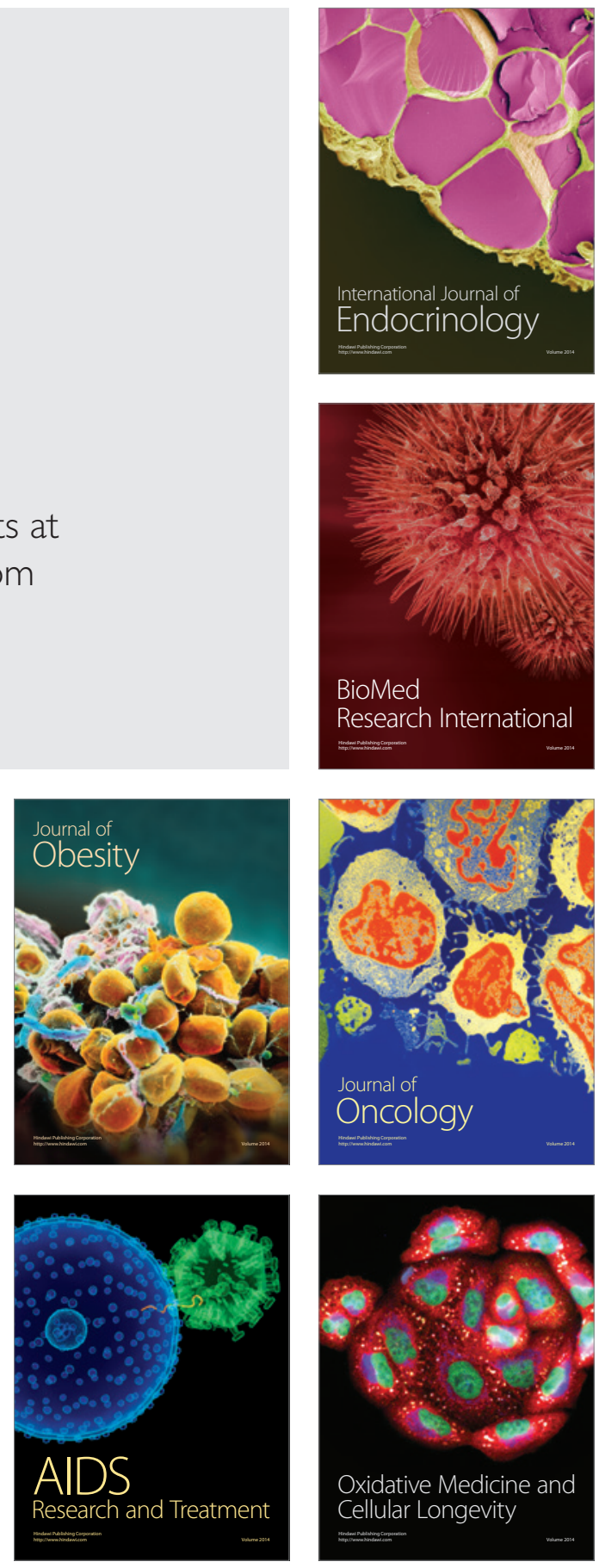